\title{
OPTIC NERVE INFILTRATION AS AN INITIAL MANIFESTATION OF T-CELL LYMPHOMA'S RELAPSE
}

\author{
Tzoukeva Al. ${ }^{1}$, A. Kaprelyan ${ }^{1}$, V. Kaleva ${ }^{2}$, D. Georgiev ${ }^{3}$, D. D. Georgiev ${ }^{3}$ \\ ${ }^{1}$ Department of Neurology, St. Marina University Hospital of Varna, \\ ${ }^{2}$ Department of Pediatrics and Medical Genetics, St. Marina University Hospital of Varna, \\ ${ }^{3}$ Dobrin Georgiev Eye Hospital of Varna
}

\begin{abstract}
Direct invasion of the orbit by the neoplastic cells from systemic T-cell non-Hodgkin's lymphoma is common. However, optic nerve involvement as an initial manifestation of relapsed disease is relatively rare. A 13-year old boy with systemic T-cell non-Hodgkin's lymphoma at stage IV in remission complained of progressive visual loss in his left eye. Ophthalmoscopy detected left optic disk edema and retinal venous engorgement. Magnetic resonance imaging showed evidence of left optic nerve head infiltration. Optical coherence tomography indicated swollen left optic nerve head. A unilateral optic nerve infiltration as an initial isolated presentation of T-cell non-Hodgkin's lymphoma relapse was diagnosed. The patient underwent systemic chemotherapy and whole brain radiotherapy of both orbits. Incomplete recovery of his left eye visual acuity was reported. Unfortunately, the child died of systemic relapse 5 months later. The rare optic nerve infiltration as an initial manifestation of T-cell lymphoma's relapse requires urgent investigation and treatment.
\end{abstract}

Key words:optic nerve infiltration, systemic T-cell non-Hodgkin's lymphoma, magnetic resonance imaging, optical coherence tomography, case report

\section{INTRODUCTION}

Central nervous system (CNS) involvement and ocular problems are becoming more common in patients with T-cell non-Hodgkin's lymphoma because of the increased survival rate due to recent treatment $(3,4,6)$. Direct invasion of the orbit by the neoplastic cells from this systemic lymphoma occurs often, however, optic nerve involvement is, typically, present in already established or recurrent lymphoma $(3,5,8)$. Isolated optic nerve infiltration as an initial relapse's manifestation in a patient with complete remission is relatively rare $(1,2,7)$. Moreover, it is relatively unaffected by systemic chemotherapy and thus, radiotherapy is often required $(9,10)$.

\section{CASE REPORT}

In November 2009, a 10-year old boy was diagnosed with systemic T-cell non-Hodgkin's lymphoma (NHL) at stage IV when he presented with intermittent high grade fever, lymphadenopathy, and splenomegaly. He was treated according to the pediatric NHL-BFM 95 protocol and a complete remission was achieved. In August 2011, he com-

\footnotetext{
Address for correspondence:

Al. Tzoukeva, Dept. of Neurology,

"Prof. Paraskev Stoyanov" Medical University of Varna,

55 M. Drinov Str., 9002 Varna, Bulgaria

e-mail: al_tz@abv.bg
}

plained of pain and progressive visual loss in his left eye. His best-corrected visual acuity was 20/20 in the right eye and 20/200 in left eye, which decreased to hand movement only within 7 days. Ophthalmoscopy detected left optic disc edema and retinal venous engorgement. Magnetic resonance imaging of brain and orbit did not show any brain pathology. There was evidence of left optic nerve head infiltration (Fig. 1). Optical coherence tomography (OCT) indicated swollen left optic nerve head (Fig. 2). Results from cerebrospinal fluid (CSF) examinations as well as from cytomorphological and flow cytometric bone marrow analyses were normal for the state of remission. A unilateral optic nerve infiltration as an initial isolated presentation of T-cell NHL relapse was diagnosed. Systemic chemotherapy and whole brain radiotherapy of both orbits were performed. After 2 months a CSF study showed pathological cells, however, an incomplete recovery of boy's left eye visual acuity 20/400 was reported. Ophthalmoscopy showed optic disc pallor. Conventional chemotherapy was delayed as boy's general status deteriorated rapidly. Unfortunately, the child died of systemic relapse 5 months later.

\section{DISCUSSION}

Optic disc changes can develop secondarily to direct infiltration by lymphomatous cells of the optic nerve head, or to swelling resulting from elevated intracranial pressure $(1-3,6-8)$. Here we reported a case of optic nerve involve- 

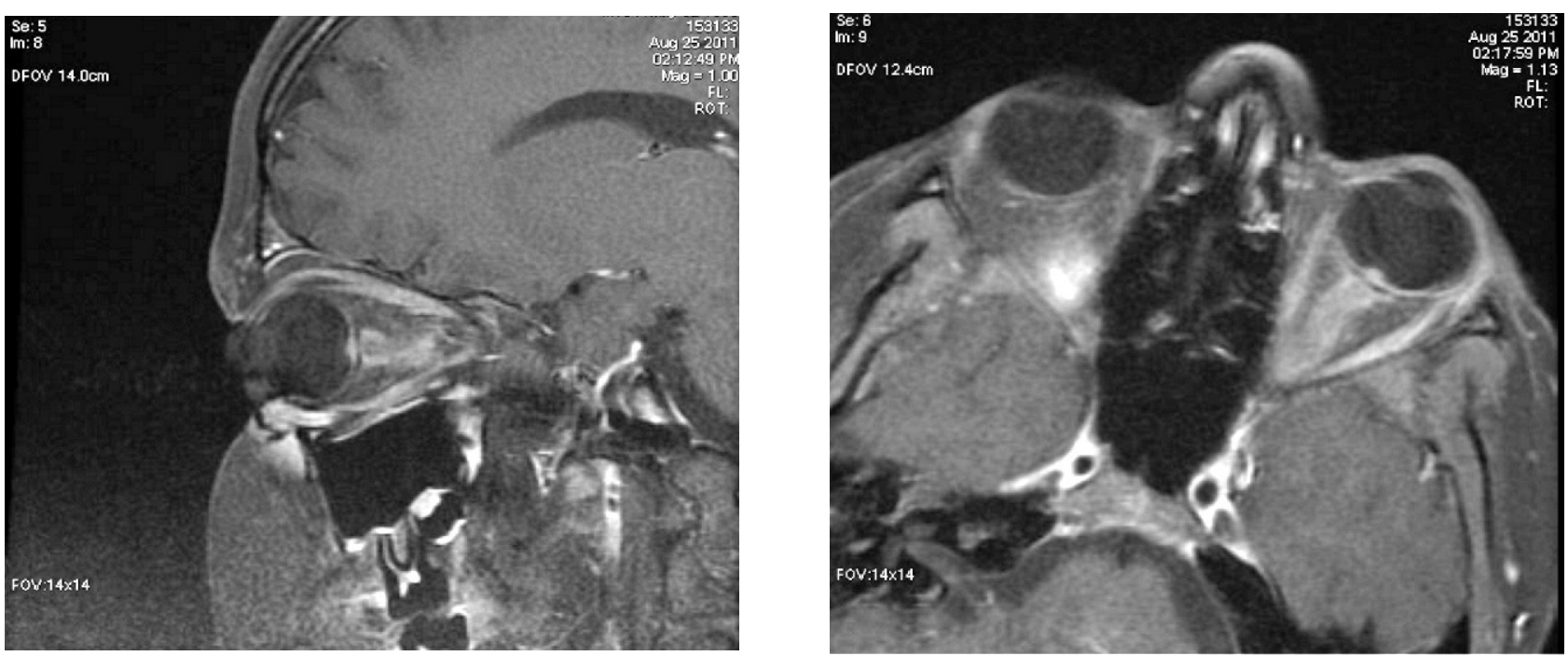

Fig. 1. MRI image of a soft-tissue component wrapping around the left optic nerve and small intrabulbar lesion compatible with lymphoma's infiltration
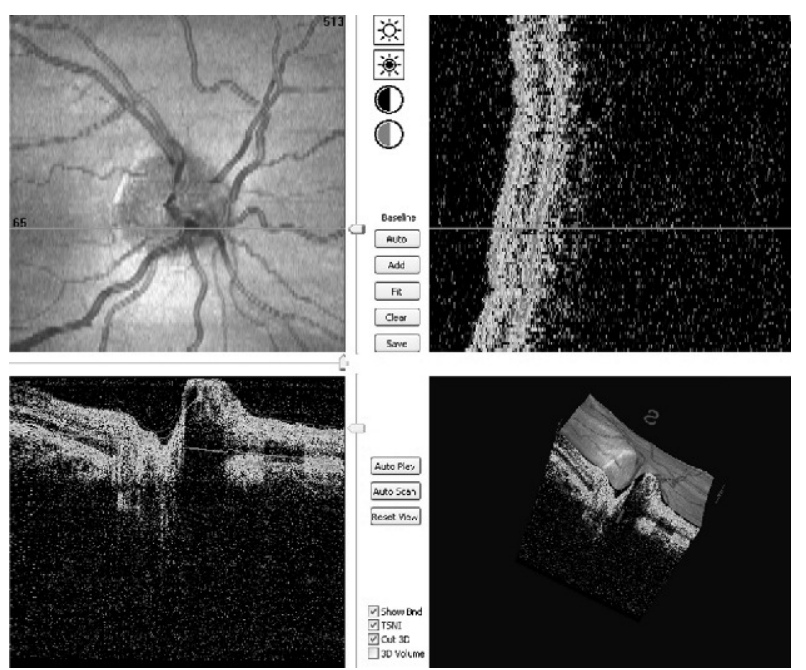
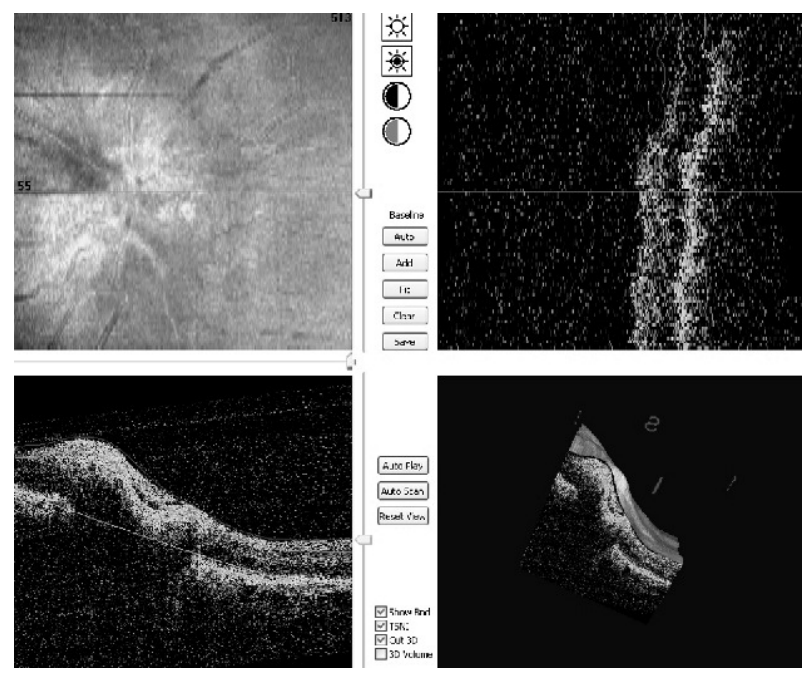

Fig. 2. OCT of optic nerve indicates swollen left optic nerve head

ment as the first evidence of CNS relapse of T-cell NHL in prolonged remission. Two questions raise after the ophthalmoscopy: if this fundoscopic evidence is a simple papilledema as a CNS brain's presentation/meningeosis, or if it is an isolated optic nerve's infiltration only. MRI and CSF examinations demonstrate that the last hypothesis is correct $(4,5)$. On the other hand, because of the fact that CSF and flow cytometry are normal as in complete remission we should have in mind boy's intolerance to chemotherapy. The problem emerged concerning the necessity to recommend the new course of treatment according to the protocol with radiotherapy in such cases. We accepted the positive answer because of CSF and flow cytometry confirmation two months later. The previous publications suggested that intrathecal chemotherapy may not be effective to retrobulbar optic nerve treatment of lymphoma's relapse $(9,10)$. In these cases, radiotherapy to the brain and orbit is recommended. However, progression to optic disc atrophy may occur despite treatment.

\section{CONCLUSION}

Optic nerve infiltration as an initial manifestation of T-cell NHL's relapse represents a visual emergency and requires urgent investigation and timely treatment. This could ensure an adequate visual recovery and, possibly, an increased patient's survival rate.

\section{REFERENCES}

1. Ali, M. J., S. G. Honavar. Optic nerve infiltration in relapse of acute lymphoblastic leukemia.Oman J. Ophthalmol., 4, 2011, No 1, p. 40. 
2. Bandyopadhyay, S., D. Das, G. Das, S. Gayen. Unilateral optic nerve infiltration as an initial site of relapse of lymphoblastic leukemia in remission.- Oman J. Ophthalmol., 3, 2010, No 3, 153-154.

3. Kitzmann, A. S., J. S. Pulido, J. A. Garrity, T. E. Witzig. Histologic finding in T-cell lymphoma infiltration of the optic nerve.- Ophthalmology, 115, 2008, No 5, e1-e6.

4. Lin, Y. C., A. G. Wang, M. Y. Yen, W. M. $\mathrm{Hsu}$. Leukaemic infiltration of the optic nerve as the initial manifestation of leukaemic relapse.- Eye, 18, 2004, No 5, 546-550.

5. Madami, A., C. Christophe, A. Ferster, B. Dan. Peri-optic nerve infiltration during leukaemic relapse: MRI diagnosis.- Pediatr. Radiol., 30, 2000, No 1, 30-32.

6. Marin, O., K. Hasui, C. Remondegui, E. Sato, M. M. Aye, N. Takenouchi, et al.
Adult T-cell leukaemia/lymphoma in Jujuy, North-West Argentina.- Pathol. Int., 52, 2002, No 5-6, 348-357.

7. Mateo, J., R. Abarzuza, E. Núñez, J. A. Cristybal. Bilateral optic nerve infiltration in acute lymphoblastic leukemia in remission.- Arch. Soc. Esp. Oftalmol., 82, 2007, No 3, 167-170.

8. Mayo, G. L., J. E. Carter, S. J. McKinnon. Bilateral optic disk edema and blindness as initial presentation of acute lymphocytic leukemia.- Am.J. Ophthalmol., 134, 2002, No 1, 141-142.

9. Shibasaki, H., S. Hayasaka, S. Noda, Y. Masaki, D. Yamamoto. Radiotherapy resolves leukemic involvement of the optic nerves.- Ann. Ophthalmol., 24, 1992, No 10, 395-397.

10. Yamamoto, N., M. Kiyosawa, T. Kawasaki, T. Miki, T. Fujino, T. Tokoro. Successfully treated optic nerve infiltration with adult T-cell lymphoma.- J. Neuroophthalmol., 14, 1994, No 2, 81-83. 